\title{
New techniques? Yes, but let it be ethical. A warning from ISCoS
}

\author{
JJ Wyndaele, Editor \\ Antwerp University Hospital, Antwerp, Belgium \\ E-mail: spinalcord@uza.be
}

Dear Spinal Cord reader,

On behalf of the International Spinal Cord Society (ISCoS), the Editorial page of this issue is devoted to a warning to spinal cord injury (SCI) individuals.

'People living with SCI are interested in any treatment that will increase their functional capabilities and improve their quality of life. Much of the excitement for therapeutic cell transplants comes from the reported benefits of cell transplants for other disorders. It is true that certain bone marrow-derived (hematopoietic) stem cells have been successfully used to treat some blood-borne and immunological disorders, but to date, this has not been the case for embryonic stem cells or other 'adult' stem cells in the treatment of SCI. Nevertheless, cell transplants into the injured spinal cord are being offered by several clinics around the world.

As with any serious medical condition, people are susceptible to claims promising recovery, even when the costs are high and potential risks are unknown. It is morally unacceptable to prey on desperate people and profit from their hope for a cure, but it happens. Please remember, if it sounds too good to be true, it is too good to be true! Individuals or institutions selling cellular transplants have not carried out controlled trials with valid methods and outcomes recorded by objective (blinded) observers. Because of a clear conflict of interest or bias, it is not acceptable that those who profit from providing a treatment should also carry out the evaluation of the treatment.

Providers of these cell transplant procedures attempt to establish credibility by citing experimental studies that have no direct relation to SCI (see above, blood-borne disorders). They also rely on reports from patients or family members. Patient-reports are tainted by what is known as the placebo effect where the patient's belief in a treatment results in their description of an improvement when nothing has actually changed. This is especially true after SCI where sensation, muscle spasms or residual reflexive movements can vary daily. The only accurate way to determine that a treatment is beneficial is to carry out a properly designed study with an appropriate control group. ISCoS believes it is unethical to charge patients for experimental interventions that are not yet proven safe or effective. Some properly conducted trials are now being undertaken, but we must await the results from these objective studies. It is also important to remember that valid clinical trials do not ask patients to pay money to participate in a trial or receive an experimental treatment.

Most cell-based treatments carry significant safety risks, many of which are common to surgery and transplantation in general, including the possibility that the cell transplant could stimulate the formation of cancerous tumors. Although high-risk therapies may be justified in people with terminal illnesses, the risk of using these therapies after SCI is not advised when individuals with SCI can achieve as good or better recovery with conventional medical care and rehabilitation training.

ISCoS does not rule out the possibility that cellular transplants may improve function and quality of life for recipients and justify the risks, but insist that the proof of benefit from cell transplants must come from a valid clinical-trial program. At this time, it is unethical to sell such unproven therapies and we do not advise patients to spend their money by paying for such treatments.

More information on questions that should be asked of someone offering a treatment of SCI is available in the free document 'Experimental Treatments for Spinal Cord Injury: What you should know', available in several languages at the International Campaign for Cures of Spinal Cord Injury Paralysis (www.campaignforcure.org).'

In this issue you will find several high level papers, a nice review on neural plasticity and functional recovery, and many original research reports. 